\title{
NON-LINEAR NETWORKS AND BOUNDARY VALUE PROBLEMS*
}

\author{
BY \\ T. A. DWYER \\ University of Dayton
}

1. Introduction. Birkhoff and Diaz [1] have given uniqueness theorems and a constructive existence proof for the solution of non-linear network problems of the type that arise in the study of hydraulic and electrical systems. In the present paper the class of problems to which this constructive technique can be applied is extended by removing or weakening some of the restrictions on the non-linear functions involved. Thus elements with more general conductivity functions may be introduced into certain branches of the network. In addition, weaker conditions on the functions governing the external influx at the nodes are given, allowing the strict Neumann problem for non-linear network problems (influx constant on prescribed nodes) to be treated by this method.

Particular application of the method is made to linear systems arising from the boundary value problems for the quasi-linear difference equation, $D U=f(U)$, where $D$ is the Laplace difference operator. A special case of the theorems for this equation yields the proof of convergence of an iterative procedure for the Neumann and mixed problems for the Laplace difference equation. The convergence of this iterative procedure for the Dirichlet problem was given by Diaz and Roberts [2].

2. Definitions and notation; the general network problem. A (finite) directed graph or digraph [3] is a finite set of $n$ nodes together with a finite set of $m$ ordered pairs of distinct nodes of the given set. The ordered pairs are called branches, and the nodes that determine a branch are called adjacent. A branch and its nodes are said to be incident on each other, and the digraph can be specified by means of its incidence matrix $\left(\epsilon_{i j}\right)$ where $\epsilon_{i j}$ is $-1,+1$, or 0 if the $i$ th node is an initial, final or non-incident node of the $j$ th branch. For the node set we write $P=\left(P_{1}, P_{2}, \cdots, P_{n}\right)$ and for the branch set $S=\left(S_{1}, S_{2}, \cdots, S_{m}\right)$.

A network $N$ is a digraph such that (i) each node is incident on at least one branch, and (ii) a non-empty proper subset of $P$ denoted by $B=\left(P_{k_{1}}, P_{k_{2}}, \cdots, P_{k_{b}}\right)$ is distinguished as the boundary of $N$. A network is called proper [4] if no more then one branch is determined by a pair of nodes. In this event, the notation $P_{a} P_{b}$ can be used without ambiguity for the corresponding directed branch. A (directed) path [3] from $P_{0}$ to $P_{k}$ consists of a collection of distinct nodes $P_{0}, P_{1}, P_{2}, \cdots, P_{k}$ together with the branches $P_{0} P_{1}, P_{1} P_{2}, \cdots, P_{k-1} P_{k}$. A network is called connected if a path exists between any two nodes of $G$. With no loss of generality we may restrict ourselves to proper connected networks.

We consider two functions whose domains are network elements. A node function $U$ is a real-valued function with domain $P$, and a brainch function $I$ is a real-valued function with domain $S$. These functions can be interpreted, for example, as the voltage

${ }^{*}$ Received October 17, 1960. The results of this paper were obtained at Case Institute of Technology in the course of research sponsored by the National Science Foundation, Grant NSF-G 9655. The author is indebted to Prof. Charles Saltzer under whose directorship this research was pursued, for his many valuable suggestions. 
and current matrices (vectors) for electrical networks [5]. The value of $U$ at $P_{i}$ will be written $U\left(P_{i}\right)$ or $U_{i}$, and the value of $I$ on $S_{i}$ will be written $I\left(S_{i}\right)$ or $I_{i}$. If $U$ is a given node function, we will write $(\delta U)_{i}$ for the difference between $U$ at the initial node of $S_{i}$ and $U$ at the final node of $S_{i}$. Corresponding to a node function $U$ it will be convenient to define a branch function $U^{*}$ having the value $(\delta U)_{i}$ on $S_{i}$.

Let $C=\left(c_{i}\right)$ be a set of real-valued functions of a real variable (conductivity functions) associated biuniquely with the oriented branches of $N$, and $G=\left(g_{i}\right)$ be a set of real-valued functions of a real variable (influx functions) associated biuniquely with the nodes of $N$. With the aid of these sets of functions we define transformations in the spaces of node and branch functions as follows. The node function

$$
Y=G(U)
$$

is defined by

$$
Y_{i}=Y\left(P_{i}\right)=g_{i}\left(U\left(P_{i}\right)\right)
$$

and the branch function

$$
J=C(I)
$$

is defined by

$$
J_{i}=J\left(S_{i}\right)=c_{i}\left(I\left(S_{i}\right)\right) .
$$

We are now in a position to state the network problem which we wish to consider.

(I) General network problem. Let $N$ be a network with node set $P$, boundary set $B$, and branch set $S$. Let $F$ be a given node function defined on $B$, and let $C=\left(c_{i}\right)$ and $G=\left(g_{i}\right)$ be given sets of real-valued functions of real variables associated biuniquely with the branches and nodes of $N$ respectively. We seek to show the existence (through the convergence of an iterative procedure) and uniqueness of a node function $U$ such that

$$
U_{i}=F_{i}, \text { for } P_{i} \varepsilon B
$$

and

$$
\sum_{i=1}^{m} \epsilon_{h i} I_{i}=Y_{h}, \text { for } P_{h} \varepsilon(P-B),
$$

where

$$
Y=G(U)
$$

and

$$
I=C\left(U^{*}\right) \text {. }
$$

Equation (2.6) will be recognized as the statement that the sum of the branch currents leaving a node is equal to the external influx of current, while (2.8) expresses the condition that the current in a given branch be a function of the potential difference across that branch. If the given influx functions are identically zero for nodes in $(P-B)$, Eq. (2.6) can be interpreted as a generalization of the discrete harmonicity condition while (2.5) becomes a statement of the boundary conditions for the problem. In this case we can speak of a "Dirichlet problem". On the other hand, if the influx functions are required only to be non-increasing continuous functions of the value of $U$ at the node 
with which they are associated, we will speak of a "mixed problem". A Neumann problem results when these functions are given as constant at each node. In this case in order to insure a unique solution, the value of $U$ must be assigned at an arbitrary node which then becomes the boundary $B$ for the problem. It will be shown in an appendix that condition (2.6) also holds at this single node of $B$, provided the given data satisfy a necessary condition.

The proof of convergence of the iterative method used in [1] is based on the following restrictions on the functions in $C$ and $G$

$$
\begin{aligned}
& c_{i}(x) \text { is a continuous function of } x, j=1,2, \cdots, m \\
& c_{j}(x) \text { is a strictly increasing function of } x, j=1,2, \cdots, m \\
& \lim _{x \rightarrow+\infty} c_{j}(x)=+\infty \text { and } \lim _{x \rightarrow-\infty} c_{j}(x)=-\infty, \quad j=1,2, \cdots, m \\
& c_{i}(0)=0, \quad j=1,2, \cdots, m \\
& g_{i}(x) \text { is a non-decreasing function of } x, i=1,2, \cdots, n .
\end{aligned}
$$

For every $g_{i}$ which does not vanish identically there exists a number $x_{1}$ such that $g_{i}\left(x_{1}\right) \leq 0$ and a number $x_{2}$ such that

$$
g_{i}\left(x_{2}\right) \geq 0, \quad i=1,2, \cdots, n \text {. }
$$

Condition (2.10) excludes branch elements with non-decreasing conductivity functions over part of their operating range (as, for example, Thyrite elements) while (2.11) excludes saturation currents (which are typical of thermionic devices). Restriction (2.12) rules out the consideration of networks with potential sources in the branches. Condition (2.14) excludes the Neumann problem since it does not permit a non-zero constant current influx at a node. We shall show that conditions (2.12) and (2.14) can be eliminated, and that there are subsets of the branch set $S$ on which (2.10) and (2.11) need not hold. In order to state the new conditions more precisely, we first introduce the concept of a dominant branch set.

Let $N$ be a network with node set $P$. We define a function $Q^{*}$ with domain $P \times P$ and range in the non-negative integers by

$$
\begin{gathered}
Q^{*}\left(P_{i}, P_{i}\right)=\min \left[\begin{array}{c}
x \mid x=\text { number of branches in a } \\
\text { path from } P_{i} \text { to } P_{i}
\end{array}\right] \\
Q^{*}\left(P_{i}, P_{i}\right)=0 .
\end{gathered}
$$

Let $B$ be a non-empty proper subset of $P$, and let $B^{*}$ be the class of all such sets. We define a function $Q$ with domain $B^{*} \times P$ and range in the non-negative integers by

$$
Q\left(B, P_{i}\right) \equiv \min _{i}\left[Q^{*}\left(P_{i}, P_{i}\right), P_{i} \varepsilon B\right] \text {. }
$$

We shall say that $P_{i}$ is equivalent to $P_{i}$ with respect to the set $B$, if $Q\left(B, P_{i}\right)=Q\left(B, P_{i}\right)$. We thus divide the set $P$ into a finite number of equivalence classes with respect to a given proper subset $B$, which we write as $K_{0}, K_{1}, \cdots, K_{a}$. The class $K_{r}$ is represented by any node $P_{i}$ such that $Q\left(B, P_{i}\right)=r$, where $r$ is called the rank of the class. Thus $K_{q}$ is the class such that $q=\max Q\left(B, P_{i}\right)$ and $K_{0}=B$. We now prove.

Lemma 2.1 Let the node set $P$ of a network $N$ with boundary $B$ be divided into 
equivalence classes with respect to $B$ as above. Let $P_{i} \varepsilon K_{r}, K_{r} \neq B$, and let $P^{*}$ be the set of nodes in $P$ adjacent to $P_{j}$. Then

$$
P^{*} \subset\left(K_{r-1} \cup K_{r} \cup K_{r+1}\right),
$$

and

$$
K_{r-1} \text { is not empty, } \quad 0<r \leq q .
$$

Proof. Let $P_{k}$ be a node adjacent to $P_{j}$. It is clear that a minimal path from $P_{k}$ to $B$ can differ from a minimal path from $P_{i}$ to $B$ by at most one segment, and hence the nodes adjacent to $P_{j}$ lie in either $K_{r-1}, K_{r}$, or $K_{r+1}$. Now consider a minimal path $W$ from $P_{i}$ to $B$. By hypothesis, $P_{i} \varepsilon K_{r}$ where $r \neq 0$, and therefore this path contains at least one branch $S_{i}$ incident on $P_{i}$. Call the other node of this branch $P_{h}$. Then the path $W$ with the branch $S_{i}$ deleted constitutes a minimal path from $P_{h}$ to $B$. (We include the possibility that this path has zero segments which would correspond to the possibility that $P_{h} \varepsilon B$.) In either case, $P_{h} \varepsilon K_{r-1}$ and therefore $K_{r-1}$ is not empty.

Definition 2.1. Let $N$ be a network with its node set $P$ divided into equivalence classes with respect to $B$, the boundary of $N$. Let $P_{i}$ be a node in $(P-B)$; thus $P_{i} \varepsilon K_{r}$, $r \neq 0$. Then as noted in the proof of Lemma 2.1 , there is at least one branch $S_{i}$ incident on $P_{j}$ such that the other node of this branch lies in an equivalence class with rank $(r-1)$. We call $S_{i}$ a dominant branch for $P_{j}$ with respect to $B$.

Definition 2.2. Let $N$ be a network with node set $P$, and boundary $B$. For each node in $(P-B)$ select a dominant branch. The set of these branches is defined as a dominant branch set of $N$ with respect to $B$, and is written as $S^{*}$.

We note that there may be more than one dominant branch per node, and that therefore $S^{*}$ is not unique. We are now in a position to state the conditions on the functions in the classes $C$ and $G$ under which we will solve the network problem (I).

Definition 2.3. Let $N$ be a network with branch set $S$ and boundary $B$. Let $S^{*}$ be a dominant branch set of $N$ with respect to $B$. Then we say that the set of conductivity functions $C=\left(c_{j}\right)$ belongs to the class $C\left(S^{*}\right)$ if, writing $c_{i}$ for the function associated with the branch $S_{i}$, we have

$$
\begin{array}{ll}
c_{j}(x) \text { is a continuous function of } x, & S_{i} \varepsilon S \\
c_{j}(x) \text { is a non-decreasing function of } x, & S_{j} \varepsilon S
\end{array}
$$

$\lim _{x \rightarrow+\infty} c_{j}(x)=+\infty$ and $\lim _{x \rightarrow-\infty} c_{j}(x)=-\infty, \quad S_{i} \varepsilon S^{*}$

$c_{i}(x)$ is a strictly increasing function of $x, \quad S_{j} \varepsilon S^{*}$.

Thus a set of conductivity functions belonging to the class $C\left(S^{*}\right)$ will have weaker conditions imposed on the functions associated with the branches of $\left(S-S^{*}\right)$ than those in [1], while condition (2.12) is eliminated completely. Since $S^{*}$ is not unique, there is some flexibility in the choice of such branches, the scope of the choice increasing with the complexity of the network

Definition 2.4. The set of functions $G=\left(g_{i}\right)$ associated with the nodes of a network $N$ will be said to belong to the class $G^{*}$ if

$$
g_{i}(x) \text { is a non-decreasing function of } x, i=1,2, \cdots, n \text {. }
$$

Our principal result will be the proof of convergence of the iterative method in [1] 
for network problems in which $C \varepsilon C\left(S^{*}\right)$ and $G \varepsilon G^{*}$. However we first prove two uniqueness theorems under somewhat weaker hypotheses.

3. Uniqueness theorems. We shall give separate theorems for the uniqueness of the branch and node functions involved in the solution of the network problem (I). The proofs of these theorems are facilitated by first establishing a network identity.

Theorem 3.1. Let $W$ be a node function and $I$ a branch function defined on a network $N$. Then

$$
\sum_{k=1}^{n} W_{k}\left(\sum_{i=1}^{m} \epsilon_{k i} I_{i}\right)+\sum_{i=1}^{m}(\delta W)_{i} I_{i}=0 .
$$

Proof. The first sum in (3.1) can be viewed as a sum of the $I_{i}$ 's with coefficients involving the $\epsilon_{k i}$ and the $W_{k}$ 's. It follows from the definition of $\epsilon_{k i}$ that if we omit terms with zero coefficients, each $I_{i}$ enters this sum twice, as $-I_{i}$ ( $W$ at initial node of $S_{i}$ ) and as $+I_{j}$ ( $W$ at final node of $S_{i}$ ), or as $-I_{j}(\delta W)_{j}$. Thus summing over all the branches of $S$, we obtain for the first sum $-\sum_{i=1}^{m}(\delta W)_{i} I_{i}$ which when added to the second sum establishes the identity.

Theorem 3.2. Let the functions in the sets $C$ and $G$ used in defining the general network problem (I) be all non-decreasing functions of a real variable, and let $U$ be a solution of this problem. Then the node and branch functions defined by $Y=G(U)$ and $I=C\left(U^{*}\right)$ are unique.

Proof. Let $U$ and $U^{\prime}$ be two different solutions of the problem and let $I=C\left(U^{*}\right)$, $I^{\prime}=C\left(U^{* \prime}\right), Y=G(U)$ and $Y^{\prime}=G\left(U^{\prime}\right)$ be the corresponding branch and node functions. Substituting the node function $\left(U-U^{\prime}\right)$ and the branch function $\left(I-I^{\prime}\right)$ in the identity (3.1), and decomposing the first sum into sums over the nodes, in $B$ and $(P-B)$, we have

$$
\begin{aligned}
& \sum_{k, B}\left[\left(U_{k}-U_{k}^{\prime}\right) \sum_{i=1}^{m} \epsilon_{k j}\left(I_{j}-I_{i}^{\prime}\right)\right]+\sum_{k,(P-B)} {\left[\left(U_{k}-U_{k}^{\prime}\right) \sum_{j=1}^{m} \epsilon_{k j}\left(I_{i}-I_{i}^{\prime}\right)\right] } \\
&+\sum_{j=1}^{m}\left[(\delta U)_{i}-\left(\delta U^{\prime}\right)_{i}\right]\left[I_{i}-I_{i}^{\prime}\right]=0,
\end{aligned}
$$

where the notation $\sum_{k, B}$, for example, indicates a sum over those values of $k$ associated with the nodes in $B$. Since $U=U^{\prime}=F$ on $B$, the first sum is zero.

From (2.6) we have that

$$
\sum_{j=1}^{m} \epsilon_{k j}\left(I_{i}-I_{i}^{\prime}\right)=\sum_{j=1}^{m} \epsilon_{k j} I_{i}-\sum_{i=1}^{m} \epsilon_{k i} I_{i}^{\prime}=Y_{k}-Y_{k}^{\prime}=g_{k}\left(U_{k}\right)-g_{h}\left(U_{k}^{\prime}\right) .
$$

Therefore, since each $g_{h}$ is non-decreasing, the second sum of (3.2) is non-negative. Since $I_{j}=c_{i}\left[(\delta U)_{i}\right]$ and $I_{i}^{\prime}=c_{j}\left[\left(\delta U^{\prime}\right)_{j}\right]$ and since the $c_{j}$ are non-decreasing, each term in the last sum of (3.2) is also non-negative and therefore zero. If the second factor of say the $k$ th term of this sum is zero, then $I_{k}=I_{k}^{\prime}$. If the first factor is zero, $(\delta U)_{i}=\left(\delta U^{\prime}\right)_{i}$ and again $I_{k}=I_{k}^{\prime}$. Thus $I=I^{\prime}$. Similarly each term of the second sum is zero, and $Y=Y^{\prime}$ which proves the theorem.

We note then, that under the hypothesis that the functions in the classes $C$ and $G$ are merely non-decreasing, we are assured a stable state of branch currents and a stable state of external influxes into the nodes. However examples can be constructed to show that several potential states (node functions $U$ ) can exist and be solutions of the network problem under this hypothesis. We shall now show that it suffices to strengthen 
the conditions on the conductivity functions associated with a dominant branch set to insure a stable potential state (unique solution $U$ ).

Theorem 3.3. Consider a general network problem (I) in which the functions in the set $G$ are non-decreasing. Let $B$ be the boundary set for this problem, and let $S^{*}$ be a dominant branch set with respect to $B$. Let the functions in the set $C$ associated with the branches of $S^{*}$ be strictly increasing, while the remaining functions (associated with the branches of $\left(S-S^{*}\right)$ ) are non-decreasing. Then if $U$ is a solution of (I) it is unique.

Proof. Assume two solutions $U$ and $U^{\prime}$ and let $I=C\left(U^{*}\right), I^{\prime}=C\left(U^{* \prime}\right), Y=G(U)$, and $Y^{\prime}=G\left(U^{\prime}\right)$ be the corresponding current and influx functions. Proceeding as in Theorem 3.2 we again obtain

$$
\begin{aligned}
\sum_{k,(P-B)}\left[U_{k}-U_{k}^{\prime}\right]\left[g_{k}\left(U_{k}\right)\right. & \left.-g_{k}\left(U_{k}^{\prime}\right)\right] \\
& +\sum_{j=1}^{m}\left\{(\delta U)_{i}-\left(\delta U^{\prime}\right)_{i}\right\}\left\{c_{i}\left[(\delta U)_{i}\right]-c_{j}\left[\left(\delta U^{\prime}\right)_{j}\right]\right\}=0 .
\end{aligned}
$$

Since all the $c_{i}$ and $g_{k}$ are at least non-decreasing, each term of (3.3) must be zero. Considering the last sum of (3.3), if $(\delta U)_{i}-\left(\delta U^{\prime}\right)_{j}$ is zero for every $j$, then $U=U^{\prime}+$ constant node function. But $U=U^{\prime}$ on $B$, and therefore $U=U^{\prime}$. Suppose now $(S U)_{k} \neq\left(\delta U^{\prime}\right)_{k}$ for some $K$. Then

$$
c_{k}\left[(\delta U)_{k}\right]=c_{k}\left[\left(\delta U^{\prime}\right)_{k}\right] .
$$

Thus in this case $I=I^{\prime}$, but $U \neq U^{\prime}$. Therefore there exists a node $P_{h}$ where $U_{h} \neq U_{h}^{\prime}$. By Lemma 2.1 and Definition 2.1 we can select a dominant branch $S_{i}$ incident on $P_{h}$ such that the other node of $S_{j}$ is in an equivalence class (with respect to $B$ ) of rank one less than that containing $P_{h}$. Then, since $c_{i}$ is a strictly increasing function on this branch, and $I_{i}=I_{i}^{\prime}$, we must have $(\delta U)_{j}=\left(\delta U^{\prime}\right)_{i}$. Therefore at the other node of this branch (call it $P_{k}$ ), $U_{k} \neq U_{k}^{\prime}$. This argument can be continued, tracing a path through nodes in equivalence classes of successively lower rank until we reach a node in $K_{0}=B$. But this contradicts $U=U^{\prime}$ on $B$ and the assumption that $(\delta U)_{k} \neq\left(\delta U^{\prime}\right)_{k}$ is impossible. Thus $U=U^{\prime}$ on $P_{0}$ (and perforce $I=I^{\prime}$ and $Y=Y^{\prime}$ ).

4. Upper and lower functions. The proofs of convergence of the iterative methods in [1], [2] and [6] are dependent upon the existence of node functions that take on the given boundary values on $B$ while they have a property that can be described as "discrete superharmonicity" or "discrete subharmonicity" on the remaining nodes of $P$. Such functions are called "upper" and "lower" functions [6] and in this section we shall show that generalized versions of such functions exist for the general network problem.

In this and the following section it will be convenient to use a more explicit notation to express the current in a branch as a function of the potential difference across the branch. We have previously indicated this relationship by writing $I_{j}=c_{j}\left[(\delta U)_{i}\right]$ for the current in the direction assigned the $j$ th branch. In writing Eq. (2.6) we imply through the use of the $\epsilon_{h i}$ that a current from node 1 to node 2 is the negative of the current from node 2 to node 1 . By writing a pair of conductivity functions $c_{i j}$ and $c_{i i}$ for the branch $P_{i} P_{i}$ we can make this assumption explicit as

$$
c_{i j}\left(U_{i}-U_{j}\right)=-c_{j i}\left(U_{i}-U_{i}\right),
$$

where $c_{i i}\left(U_{i}-U_{i}\right)=I_{i i}$ gives the current from node $P_{i}$ to $P_{i}$ and $c_{i i}\left(U_{i}-U_{i}\right)=I_{i i}$ 
gives the current from node $P_{j}$ to $P_{i}$, where $P_{i}$ and $P_{j}$ are the end nodes of the branch in question, but not designated as initial or final. Thus (2.6) becomes

$$
\sum_{i=1}^{n} c_{i h}\left(U_{i}-U_{h}\right)=Y_{h}
$$

where terms in the sum on the left are taken as zero for those values of $j$ for which the branch $P_{i} P_{h}$ does not exist.

Definition 4.1. Let $N$ be a network with a set of $2 m$ conductivity functions $C=\left(c_{i i}\right)$ associated with the branch set $S$ (the pair of functions $c_{i i}$ and $c_{i i}$ corresponding to the branch $\left.P_{i} P_{i}\right)$ and with a set of $n$ influx functions $G=\left(g_{i}\right)$ associated with the node set $P$. Then a node function $U$ defined on $P$ is said to be superformed with respect to $C$ and $G$ at the node $P_{i}$ if

$$
\sum_{i=1}^{n} c_{i i}\left(U_{i}-U_{i}\right) \leq Y_{i} \text { where } Y=G(U) .
$$

In an analogous manner, we say that $U$ is subformed with respect to $C$ and $G$ at $P_{i}$ if

$$
\sum_{i=1}^{m} c_{i i}\left(U_{i}-U_{i}\right) \geq Y_{i} \text { where } Y=G(U) .
$$

Definition 4.2. Let $N$ be a network with associated sets of $2 m$ conductivity functions $C=\left(c_{i j}\right)$ and $n$ influx functions $G=\left(g_{i}\right)$ as above, and let $F$ be a node function defined on the boundary of the network $B$. We then say that a node function $W$ defined on the node set $P$ of $N$ is an upper function on $N$ with respect to $C, G$, and $F$ if

$$
W_{k}=F_{k}, \quad P_{k} \varepsilon B,
$$

$W$ is superformed with respect to $C$ and $G$ at all $P_{i} \varepsilon(P-B)$.

Definition 4.3. In an analogous manner we say that a node function $V$ defined on $P$ is a lower function on $N$ with respect to $C, G$, and $F$ if

$$
V_{k}=F_{k}, \quad P_{k} \varepsilon B,
$$

$V$ is subformed with respect to $C$ and $G$ at all $P_{i} \varepsilon(P-B)$.

Lemma 4.1. Using the notation of Definition 4.2, let $W$ be an upper function on $N$ with respect to $C, G$, and $F$ where $G \varepsilon G^{*}$. Let $M$ be a node function defined on $P$ by

(i) $\quad M_{k_{i}}=m_{k_{i}}$ for $P_{k_{i}} \varepsilon B$ where $\left(m_{k_{1}}, m_{k_{2}}, \cdots, m_{k_{b}}\right)$ is a set of $b$ non-negative constants.

(ii) $\quad M_{k}=\left(\max \left(m_{j}\right), \quad j=k_{1}, k_{2}, \cdots, k_{b}\right)+A$

for $P_{k} \varepsilon(P-B)$, where $A$ is a non-negative constant.

Let each $c_{i j}(x)$ be a non-decreasing function of $x$. Then the node function $Z=W+M$ is an upper function with respect to $C, G$, and $F^{\prime}$, where $F^{\prime}$ is the node function defined on $B$ by $F_{j}^{\prime}=F_{i}+m_{i}, j=k_{1}, k_{2}, \cdots k_{b}$.

Proof. It is clear that the only branches on which $\left(Z_{i}-Z_{k}\right)$ differs from $\left(W_{i}-W_{k}\right)$, 
where $P_{i}$ and $P_{k}$ are the end nodes of such branches are those incident on nodes of $B$. But here if we write $P_{i}$ for a node in $B$ and $P_{k}$ for an adjacent node not in $B$, we have

$$
\begin{aligned}
Z_{i}-Z_{k} & =\left(W_{i}+m_{i}\right)-\left(W_{k}+\max _{i}\left(m_{i}\right)+A\right) \\
& \leq W_{i}+m_{i}-W_{k}-m_{i}=W_{i}-W_{k} .
\end{aligned}
$$

Thus, since the functions in $C$ are non-decreasing, we have

$$
\sum_{i=1}^{n} c_{i k}\left(Z_{i}-Z_{k}\right) \leq \sum_{i=1}^{n} c_{i k}\left(W_{i}-W_{k}\right) \leq G\left(W_{k}\right) \leq G\left(Z_{k}\right) .
$$

Thus, since $Z_{i}=\left(W_{i}+m_{i}\right)=\left(F_{i}+m_{i}\right)=F_{i}^{\prime}$ for $P_{i} \varepsilon B, Z$ is an upper function with respect to $C, G$, and $F^{\prime}$.

We now show that the weakened restrictions on the function sets $C$ and $G$ given by (2.20), (2.21), (2.22), (2.23), and (2.24) are sufficient to guarantee the existence of upper and lower functions for arbitrary $F$ and $B$ by indicating how such functions can be constructed.

Theorem 4.1. Let $N$ be a network with node set $P$, branch set $S$, and boundary set $B$. Let $S^{*}$ be a dominant branch set of $N$ with respect to $B$, and let $C$ be a set of conductivity functions in the class $C\left(S^{*}\right)$. Let $G$ be a set of finite valued influx functions, and let $F$ be a node function defined on $B$. Then there exists a node function $W$ defined on $P$ such that $W$ is an upper function on $N$ with respect to $C, G$, and $F$.

Proof. We proceed by showing how such a function $W$ can be constructed. Define

$$
W_{i}=F_{i} \text { for } P_{i} \varepsilon B \text {. }
$$

Now consider the nodes in $K_{a}$, where $K_{a}$ is the equivalence class in $P$ of highest order. Since $B$ is a proper subset of $P, q \neq 0$. Define

$$
W_{i}=a \text { for } P_{i} \varepsilon K_{a}, \quad \text { where } a \text { is a real parameter. }
$$

By Lemma 2.1, incident on any node $P_{j}$ in $K_{q}$ there is a dominant branch $P_{k} P_{j}$ such that $P_{k}$ is in $K_{a-1}$. From the definition of $C\left(S^{*}\right), \lim _{x \rightarrow-\infty} c_{k j}(x)=-\infty$. Thus for each $P_{i} \varepsilon K_{\varepsilon}$, and for any two real numbers $Z_{0}$ and $U_{0}$ there exists a positive $d_{i}^{\prime}$ such that

$$
c_{k i}\left(-d_{i}^{\prime}+Z_{0}\right) \leq-\sum_{\substack{h \\ h \neq k}} c_{h i}\left(Z_{0}\right)+g_{i}\left(U_{0}\right) .
$$

In particular, letting $Z_{0}=0$, and $U_{0}=a$, we have

$$
c_{k i}\left(-d_{i}^{\prime}\right) \leq-\sum_{\substack{h=1 \\ h \ngtr k}}^{n} c_{h i}(0)+g_{i}(a)
$$

Let

$$
d_{a}=\max \left(d_{i}^{\prime}, P_{i} \varepsilon K_{\imath}\right)
$$

Now define

$$
W_{k}=\left(a-d_{a}\right), \text { for } P_{k} \varepsilon K_{a-1} .
$$

Thus by (4.10), (4.12), (4.13), and (4.14) for any node $P_{i}$ in $K_{a}$ there exists a node $P_{k}$ adjacent to $P_{i}$ such that 


$$
\begin{aligned}
c_{k i}\left(W_{k}-W_{j}\right) & =c_{k i}\left(a-d_{a}-a\right)=c_{k j}\left(-d_{q}\right) \\
& \leq c_{k j}\left(-d_{i}^{\prime}\right) \leq-\sum_{\substack{h \\
h \neq k}} c_{h i}(0)+g_{i}(a) .
\end{aligned}
$$

But by Lemma 2.1, for $P_{h}$ adjacent to a node $P_{i}$ in $K_{e}\left(W_{h}-W_{i}\right)$ has the value zero or $-\mathrm{d}_{a}$. Since each $c_{h i}$ is a non-decreasing function of its argument we therefore have

$$
-c_{h j}\left(W_{h}-W_{j}\right) \geq-c_{h j}(0)
$$

so that substituting in (4.15) we have

$$
c_{k j}\left(W_{k}-W_{i}\right) \leq-\sum_{\substack{h=1 \\ h \neq k}}^{n} c_{h j}\left(W_{h}-W_{i}\right)+g_{j}(a) .
$$

Transposing and using (4.10) we have

$$
\sum_{h=1}^{n} c_{h i}\left(W_{h}-W_{i}\right) \leq g_{j}\left(W_{i}\right)
$$

which is the statement that $W$ is superformed with respect to $C$ and $G$ at the node $P_{i}$.

Now consider any node $P_{k} \varepsilon K_{a-1}$. By Lemma 2.1 and Definition 2.1 there is a branch $P_{i} P_{k}$ in $S^{*}$ incident on $P_{k}$ such that $P_{i} \varepsilon K_{a-2}$. Thus, since $c_{i k}$ belongs to the class $C\left(S^{*}\right)$ (4.11) applies. Setting $Z_{0}=d_{a}, U_{0}=\left(a-d_{a}\right)$, we have assured the existence of a $d_{k}^{\prime \prime}>0$ such that, for each $P_{k} \varepsilon K_{a-1}$

$$
c_{i k}\left(-d_{k}^{\prime \prime}+d_{a}\right) \leq-\sum_{\substack{h \\ h \rightarrow i}} c_{h k}\left(d_{a}\right)+g_{k}\left(a-d_{a}\right) .
$$

Let

$$
d_{q-1}=\max \left(d_{k}^{\prime \prime}, P_{k} \varepsilon K_{q-1}\right)
$$

Now define

$$
W_{i}=\left(a-d_{a-1}\right) \text { for } P_{i} \varepsilon K_{q-2} \text {. }
$$

Thus for the node $P_{k} \varepsilon K_{a-1}$, we have from (4.19), (4.20), and (4.21)

$$
\begin{aligned}
c_{i k}\left(w_{i}-w_{k}\right) & =c_{i k}\left[\left(a-d_{a-1}\right)-\left(a-d_{a}\right)\right] \\
& =c_{i k}\left(-d_{a-1}+d_{a}\right) \leq c_{i k}\left(-d_{k}^{\prime \prime}+d_{a}\right) \leq \sum_{\substack{h=1 \\
h \neq k}}^{n} c_{h k}\left(d_{a}\right)+g_{i}\left(a-d_{a}\right) .
\end{aligned}
$$

But by Lemma 2.1, the nodes $P_{h}$ adjacent to $P_{k}$ (which is in $K_{a-1}$ ) lie in $K_{a} \cup K_{a-1} \cup K_{a-2}$. Thus

$$
W_{h}-W_{k}= \begin{cases}\left(a-d_{a-1}\right)-\left(a-d_{a}\right)=d_{a}-d_{a-1} & \text { or } \\ \left(a-d_{a}\right)-\left(a-d_{a}\right)=0 & \text { or } \\ a-\left(a-d_{a}\right)=d_{a} . & \end{cases}
$$

Thus, surely

$$
W_{h}-W_{k} \leq d_{a}
$$

And since all the $c_{i j}$ are non-decreasing,

$$
-c_{h k}\left(W_{h}-W_{k}\right) \geq-c_{h k}\left(d_{q}\right) \text {. }
$$


Thus, from (4.22), (4.25), and (4.21)

$$
c_{i k}\left(W_{i}-W_{k}\right) \leq-\sum_{\substack{h=1 \\ h \neq i}}^{n} c_{h k}\left(W_{h}-W_{k}\right)+g_{k}\left(a-d_{a}\right) .
$$

Thus at $P_{k}$

$$
\sum_{h=1}^{n} c_{h k}\left(W_{h}-W_{k}\right) \leq g_{k}\left(a-d_{a}\right)=g_{k}\left(W_{k}\right)
$$

which means that $W$ is superformed at $P_{k}$ with respect to $C$ and $G$. Continuing in this way we can define $W$ on each equivalence class so that it will be superformed with respect to $C$ and $G$ at each node of $K_{a}, K_{q-1}, \cdots, K_{3}, K_{2}$.

Our definition of $W$ may be summarized as follows:

$$
\begin{aligned}
& W_{i}=a \quad, \quad P_{i} \varepsilon K_{a} \\
& W_{i}=a-d_{a} \quad P_{i} \varepsilon K_{a-1} \\
& W_{i}=a-d_{a-1}, \quad P_{i} \varepsilon K_{a-2} \\
& \text {........................... } \\
& W_{i}=a-d_{2} \quad, \quad P_{i} \varepsilon K_{1} \\
& W_{i}=F_{i} \quad, \quad P_{i} \varepsilon K_{0}=B .
\end{aligned}
$$

We could in fact continue this sequence of constructions to define a constant $d_{1}$ exactly as above. Using this constant we can now define the parameter $a$ to insure that $W$ is superformed at nodes in $K_{1}$. Let

$$
a=F^{*}+d_{1},
$$

where $F^{*}=\max _{i}\left(F_{i}\right)$. But if $P_{k}$ is a node in $K_{1}$, then there is a dominant branch $P_{k} P_{h}$ in $S^{*}$ such that $P_{h}$ is in $K_{0}=B$, and

$$
W_{h}-W_{k}=F_{h}-\left(a-d_{2}\right) \leq F^{*}-\left(a-d_{2}\right)=d_{2}-d_{1} \text {. }
$$

Thus we can proceed (as in (4.19), (4.22), and (4.23)) to establish that $W$ is superformed at $P_{k} \varepsilon K_{1}$ with respect to $C$ and $G$. Thus $W$ is an upper function on $N$ with respect to $C, G$, and $F$.

Theorem 4.2. Under the same hypothesis as in Theorem 4.1 there exists a node function $V$ defined on $P$ such that $V$ is a lower function on $G$ with respect to $C, G$, and $F$.

The proof is completely analogous to that of Theorem 4.1.

5. Convergence of an iterative procedure for the non-linear network problem. We now make use of the knowledge that upper and lower functions can always be found for the network problem (I) under the hypotheses of Theorem 4.1 to show that under these same hypotheses a procedure analogous to Gauss-Seidel iteration converges for an arbitrary initial node function to the unique solution of (I).

Definition 5.1. Let $U$ be a node function defined on the node set of $P$ of a network $N$, and let $C$ and $G$ be the conductivity and influx function sets for $N$. We define the residual of $U$ at $P_{j}$ with respect to $C$ and $G$ to be

$$
R\left(U_{i}\right)=\sum_{i=1}^{n} c_{i i}\left(U_{i}-U_{i}\right)-g_{i}\left(U_{i}\right)
$$


where $R$ is viewed as a function of the single real variable $U_{i}$, all the other $U_{i}$ being held fixed.

We remark that a node function $W$ is superformed at $P_{i}$ with respect to $C$ and $G$ if and only if $R\left(W_{i}\right) \leq 0$, and a node function $V$ is subformed at $P_{i}$ with respect to $C$ and $G$ if and only if $R\left(V_{i}\right) \geq 0$.

Lemma 5.1. Let $N$ be a network with boundary $B$ and let $S^{*}$ be a dominant branch set of $N$ with respect to $B$. Let $C$ be a set of conductivity functions belonging to the class $C\left(S^{*}\right)$ and let $G$ be a set of influx functions belonging to the class $G^{*}$. Then for a node function $U$ defined arbitrarily on $\left(P-P_{i}\right)$ there is exactly one value $U_{j}$ such that

$$
R\left(U_{j}\right)=0 .
$$

Proof. Since the $U_{i}$ adjacent to $U_{i}$ are fixed, by the definition of $C\left(S^{*}\right)$ each $c_{i j}$ is a continuous non-decreasing function of $\left(-U_{i}\right)$. By the definition of $G^{*}$ each $g_{i}\left(U_{i}\right)$ is a continuous non-decreasing function of $U_{i}$ or a continuous non-increasing function of $\left(-U_{i}\right)$. But also by the definition of $C\left(S^{*}\right)$ at least one of the $c_{i j}$ (corresponding to a dominant branch incident on $\left.P_{i}\right)$ is a strictly increasing function of $\left(-U_{i}\right)$ such that

$$
\lim _{-U_{j} \rightarrow-\infty} c_{i j}\left(U_{i}-U_{j}\right)=-\infty
$$

and

$$
\lim _{-U_{i} \rightarrow+\infty} c_{i i}\left(U_{i}-U_{j}\right)=+\infty .
$$

Hence $R$ is a strictly increasing continuous function of $\left(-U_{i}\right)$ which varies continuously from $-\infty$ to $+\infty$ as $\left(-U_{i}\right)$ does, and therefore there is exactly one value of $-U_{i}$ (or $U_{j}$ ) such that $R\left(U_{i}\right)=0$.

Definition 5.2. Let $U$ be a node function defined on $P$, the node set of a network $N$, and let $C$ and $G$ be the conductivity and influx function sets for $N$. Then the node function $U^{\prime}$ is called the relaxation of $U$ at $P_{k}$ with respect to $C$ and $G$ if $(i) U_{i}^{\prime}=U_{i}, j \neq K$ and (ii) $U_{k}^{\prime}$ is the unique solution of $R\left(U_{k}\right)=0$.

In what follows we write $W \geq U$ or $W \leq U$ for two node functions if and only if the corresponding inequality holds for the values of the functions at each node.

Lemma 5.2. Let $W$ and $U$ be node functions defined on the node set $P$ of a network $N$ such that $W \geq U$. Let the set of conductivity functions for the network $C$ be in $C\left(S^{*}\right)$ and the set of influx functions $G$ be in $G^{*}$. Then if $W^{\prime}$ and $U^{\prime}$ are the relaxations of $W$ and $U$ at $P_{h}$ with respect to $C$ and $G, W^{\prime} \geq U^{\prime}$.

Proof. Suppose the lemma false. Since $W_{i}^{\prime} \geq U_{i}^{\prime}$ for $P_{i} \varepsilon\left(P-P_{h}\right)$, we must have

$$
W_{h}^{\prime}<U_{h}^{\prime} \text {. }
$$

But by Definition 5.2

$$
\sum_{i=1}^{n} c_{i h}\left(W_{i}-W_{h}^{\prime}\right)=g_{h}\left(W_{h}^{\prime}\right)
$$

and

$$
\sum_{i=1}^{n} c_{i h}\left(U_{i}-U_{h}^{\prime}\right)=g_{h}\left(U_{h}^{\prime}\right)
$$

By hypothesis $W_{i} \geq U_{i}$. From (5.3) $-W_{h}^{\prime}>-U^{\prime}$ and therefore

$$
\left(W_{i}-W_{h}^{\prime}\right)>\left(U_{i}-U_{h}^{\prime}\right) .
$$


Since by Lemma 2.1 at least one of the functions $c_{i h}$ is associated with a dominant branch, $\sum_{i} c_{i h}(x)$ is a strictly increasing function of $x$. Thus from (5.6)

$$
\sum_{i=1}^{n} c_{i h}\left(W_{i}-W_{h}^{\prime}\right)>\sum_{i=1}^{n} c_{i h}\left(U_{i}-U_{h}^{\prime}\right)
$$

and therefore by (5.4), (5.5), and (5.7)

$$
g_{h}\left(W_{h}^{\prime}\right)>g_{h}\left(U_{h}^{\prime}\right) .
$$

But from (5.3) and the hypothesis that $g_{h}(x)$ is a non-decreasing function of $x$

$$
g_{h}\left(W_{h}^{\prime}\right) \leq g_{h}\left(U_{h}^{\prime}\right)
$$

which contradicts (5.8).

Lemma 5.3. Using the same notation as in Lemma 5.2, if $W \leq U$, then $W^{\prime} \leq U^{\prime}$. The proof is analogous to that used in Lemma 5.2.

Definition 5.3. Let $N$ be a network with node set $P$ and boundary set $B$. Let $F$ be a node function defined on $B$. Then a trial function on $P$ with respect to $F$ is a node function $U$ defined on $P$ such that $U_{i}=F_{i}$ for $P_{i}$ \& $B$.

Theorem 5.1. Let $G$ be a network with node set $P, F$ a node function defined on the boundary $B, G$ a set of $n$ finite valued influx functions, and $S^{*}$ a dominant branch set of $N$ with respect to $B$. Let $C$ be a set of conductivity functions belonging to $C\left(S^{*}\right)$. Then for any trial function $U$ on $P$ with respect to $F$, there are two node functions $V$ and $W$ defined on $P$ such that

(i) $W$ is an upper function with respect to $C, G$, and $F$

(ii) $V$ is a lower function with respect to $C, G$, and $F$

(iii) $W \geq U \geq V$

Proof. By Theorem 4.1 there exists an upper function $W$ with respect to $C, G$, and $F$ and $W_{k}=U_{k}=F_{k}$ for $P_{k} \varepsilon B$. Let $M=\max \left(U_{i}, i=1,2, \cdots, n\right)$. Using the notation of Theorem 4.1 we consider two possibilities.

Case I. If $M \leq\left(a-d_{2}\right)$, then $U \leq W$ on $P$, since $\left(a-d_{2}\right)$ is the minimum value of $W$ on $(P-B)$.

Case II. If $M>\left(a-d_{2}\right)$, let $D \equiv\left(M-a+d_{2}\right)$ and define a node function $W^{*}$ by

$$
\begin{array}{rlrl}
W_{i}^{*} & =F_{i} \quad, \quad & P_{i} \varepsilon B, \\
& =W_{i}+D, & & P_{i} \varepsilon(P-B) .
\end{array}
$$

By Lemma $4.1 W^{*}$ is again an upper function with respect to $C, G$, and $F$. Further, for $P_{i} \varepsilon(P-B)$ and $P_{i} \varepsilon K_{1}$ we have

$$
W^{*}\left(P_{i}\right) \geq W^{*}\left(P_{i}\right)=W_{i}+D=\left(a-d_{2}\right)+\left(M-a+d_{2}\right) .
$$

Therefore

$$
W_{i}^{*} \geq M \geq U_{i} .
$$

Thus in either case we have a function which satisfies (i) and (iii). Similarly a function $V$ can be found satisfying (ii) and (iii).

Corollary 5.1. If $U$ is a trial function on $P$ with respect to $F$, there exist two other trial functions $V$ and $W$ (with respect to $F$ ) such that

$$
W \geq U \geq V \text { on } P \text {, }
$$


and

$$
R\left(W_{i}\right) \leq 0 \leq R\left(V_{i}\right), \quad P_{i} \varepsilon(P-B) .
$$

The proof follows from Theorem 5.1 and the remark following Definition 5.1.

Lemma 5.4. Consider a network $N$ with boundary $B$, and dominant branch set with respect to $B, S^{*}$. Let $F$ be a node function defined on $B, C$ a set of conductivity functions in $C\left(S^{*}\right), Y$ a set of influx functions in $Y^{*}$ and let $U^{\prime}$ be an upper (lower) function with respect to $C, G$, and $F$. Let $U$ be the relaxation of $U^{\prime}$ at $P_{\theta} \varepsilon(P-B)$ with respect to $C$ and $G$. Then $U$ is an upper (lower) function on $G$ with respect to $C$, $G$, and $F$.

Proof. Since $U$ differs from $U^{\prime}$ only at a node of $(P-B), U_{h}=U_{h}^{\prime}=F_{h}$ for $P_{h} \varepsilon B$. It remains to show that $U$ is superformed with respect to $C$ and $G$ at all $P_{i} \varepsilon(P-B)$. We need only consider $P_{\theta}$ and the nodes adjacent to $P_{\theta}$, since at other nodes there will have been no changes in the variables in Eq. (4.3) which defines the concept of superformed at a node. By the definition of relaxation at a node, at $P_{\theta}$

$$
\begin{gathered}
R\left(U_{\theta}\right)=0=\sum_{i=1}^{n} c_{i \theta}\left(U_{i}-U_{\theta}\right)-g_{\theta}\left(U_{\theta}\right), \\
\sum_{i=1}^{n} c_{i \theta}\left(U_{i}-U_{\theta}\right)=g_{\theta}\left(U_{\theta}\right),
\end{gathered}
$$

and thus $U$ is superformed with respect to $C$ and $Y$ at $P_{\theta}$. Consider now a node adjacent to $P_{\theta}$. In the proof of Lemma 5.1 it was shown that $R$ is a strictly decreasing function of its argument. Since $R\left(U_{\theta}^{\prime}\right) \leq 0$ while $R\left(U_{\theta}\right)=0$, it follows that

$$
U_{\theta} \leq U_{\theta}^{\prime} \text {. }
$$

Thus, since all the $c_{i j}$ are non-decreasing functions,

$$
c_{\theta i}\left(U_{\theta}-U_{i}\right) \leq c_{\theta i}\left(U_{\theta}^{\prime}-U_{i}\right) .
$$

Since $U_{i}=U_{i}^{\prime}$ if $i \neq \theta$, we have

$$
R\left(U_{i}^{\prime}\right)=\sum_{\substack{i=1 \\ i \neq \theta}}^{n} c_{i j}\left(U_{i}-U_{i}\right)+c_{\theta j}\left(U_{\theta}^{\prime}-U_{i}\right)-g_{j}\left(U_{i}\right) .
$$

Thus from (5.14), (5.12), and the hypothesis that $R\left(U_{i}^{\prime}\right) \leq 0$, we have

$$
R\left(U_{i}\right) \leq R\left(U_{i}^{\prime}\right) \leq 0 .
$$

Thus by the remark following Definition $5.1 U$ is superformed with respect to $C$ and $G$ at all nodes in $(P-B)$ and is therefore an upper function on $N$ with respect to $C, G$, and $F$. The proof for lower functions is carried out in an analogous manner.

Lemma 5.5. Using the notation of Lemma 5.4, if $U^{\prime}$ is an upper function on $N$ with respect to $C, G$, and $F$, and $U$ is the relaxation of $U^{\prime}$ at $P_{k} \varepsilon(P-B)$ with respect to $C$ and $G$, then $U \leq U^{\prime}$. Similarly, if $V^{\prime}$ is a lower function on $N$ with respect to $C, G$, and $F$, and $V$ is the relaxation of $V^{\prime}$ at $P_{k} \varepsilon(P-B)$ with respect to $C$ and $G$, then $V \geq V^{\prime}$. The proof follows from (5.13) and the analogous relation for lower functions.

We are now in a position to prove the existence of a solution of the network problem (I) under the weakened conditions on $C$ and $G$. We first define the iterative procedure on which this proof is based. 
Definition 5.3. Let $N$ be a network with node set $P$ and boundary $B$, and let $F$ be a node function defined on $B$. Let $C$ be a set of conductivity functions for $N$ in $C\left(S^{*}\right)$, and where $S^{*}$ is a dominant branch set of $N$ with respect to $B$, and let $G$ be a set of influx functions for $N$ in $Y^{*}$. Let the nodes of $(P-B)$ be arranged in a sequence $\left\{P_{i}\right\}$ with the property that each node appears infinitely often, but that the same node never appears in the sequence more than a finite number of times in succession. Then for a trial function $U^{0}$ on $P$ with respect to $F$ we define a relaxation sequence for $U^{0}$ with respect to $\left\{P_{i}\right\}$, written $\left\{U^{i}\right\}$, by

$U^{0}$ is the given trial function
$U^{1}$ is the relaxation of $U^{0}$ at the first node in $\left\{P_{i}\right\}$
with respect to $C$ and $G$
$U^{2}$ is the relaxation of $U^{1}$ at the second node in $\left\{P_{i}\right\}$
with respect to $C$ and $G$

Theorem 5.2. Using the notation of Definition 5.3 which is also the notation for the general network problem (I), the relaxation sequences $\left\{U^{i}\right\}$ for an arbitrary trial function $U^{0}$ converges to a unique node function $U$ which is the solution of the general network problem (I) for $C_{\varepsilon} C\left(S^{*}\right)$ and $G \varepsilon G^{*}$.

Proof. By Theorem 5.1 there exist trial functions $V^{0}$ and $W^{0}$ such that $V^{0}$ is a lower function with respect to $C, G$, and $F, W^{0}$ an upper function, and

$$
V^{0} \leq U^{0} \leq W^{0}
$$

Forming the relaxations of all three trial functions at the first node of the sequence $\left\{P_{i}\right\}$ we have by Lemmas 5.2 and 5.3

$$
V^{1} \leq U^{1} \leq W^{1} \text { on } P
$$

and by Lemma 5.4

$$
\begin{aligned}
& V^{1} \text { is a lower function with respect to } C, G \text {, and } F \text {. } \\
& W^{1} \text { is an upper function with respect to } C, G \text {, and } F .
\end{aligned}
$$

By Lemma 5.5 and (5.19)

$$
V^{0} \leq V^{1} \leq U^{1} \leq W^{1} \leq W^{0}
$$

Because of (5.19) and (5.20) we may repeat this argument, and after forming the relaxations of the successive functions at $n$ of the nodes in the sequence $\left\{P_{i}\right\}$ we have

$$
V^{0} \leq V^{1} \leq \cdots \leq V^{n} \leq U^{n} \leq W^{n} \leq \cdots \leq W^{1} \leq W^{0}
$$

with the assurance that, for $i=1,2, \cdots, n$

$$
\begin{aligned}
& V^{i} \text { is a lower function with respect to } C, G \text {, and } F . \\
& W^{i} \text { is an upper function with respect to } C, G \text {, and } F .
\end{aligned}
$$

From (5.22) we can see that at each node $P_{h}$ in $(P-B)$, the sequence $\left\{V^{i}\left(P_{h}\right)\right\}$ is nondecreasing and bounded above and that therefore the limit 


$$
V_{h}=\lim _{n \rightarrow \infty} V_{h}^{n}
$$

exists for $P_{A} \varepsilon(P-B)$. Define the node function $V$ to have the value

$$
V_{h} \text { for } P_{h} \varepsilon(P-B) \text { and } F_{k} \text { for } P_{k} \varepsilon B \text {. }
$$

We will show that $V$ is the solution of the network problem (I). By its definition $U$ satisfies (2.5). To show that (2.6) holds, we will show that $R\left(V_{k}\right)=0$ for $P_{k} \varepsilon(P-B)$. Let $P_{k} \varepsilon(P-B)$. Then $P_{k}$ occurs infinitely many times in the sequence $\left\{P_{i}\right\}$, and therefore there is a sequence of positive integers $n_{1}, n_{2}, \cdots$ such that

$$
R\left(V_{k}^{n_{i}}\right)=0, \quad j=1,2, \cdots
$$

Since the limit of the original sequence of trial functions exists,

$$
\lim _{n \rightarrow \infty} V^{n}\left(P_{k}\right)=\lim _{j \rightarrow \infty} V^{n i}\left(P_{k}\right) .
$$

Thus, since $R(x)$ is a continuous function of $x$,

$$
R\left[V\left(P_{k}\right)\right]=R\left[\lim _{n \rightarrow \infty} V^{n}\left(P_{k}\right)\right]=R\left[\lim _{i \rightarrow \infty} V^{n_{i}}\left(P_{k}\right)\right]=\lim _{i \rightarrow \infty} R\left[V^{n_{i}}\left(P_{k}\right)\right]=0
$$

which in view of the definition of $R\left(V_{k}\right)$ given by (5.1) establishes (4.2) which is equivalent to (2.6). Thus $V$ is a solution of problem (I). In a similar manner the sequence $\left\{W^{n}\left(P_{h}\right)\right\}$ (which is non-increasing and bounded below) is used to define a function $W$ which is by the same reasoning used for $V$ a solution of problem (I). The uniqueness result of Theorem 3.3 then assures us that $V=W$, and the inequality (5.22) shows that $U^{n}$ also converges to the unique solution of the general network problem (I).

6. Application to a quasi-linear difference equation. If we choose each of the functions $c_{j}(x)$ to be the linear function $x$, then Eq. (2.6) (or its equivalent form (4.2)) becomes

$$
\sum_{i=1}^{n}\left(U_{i}-U_{i}\right)=g_{j}\left(U_{i}\right)
$$

which may be regarded as the difference equation approximating the partial differential equation $\nabla U=g(U)$ on an arbitrary polygonal grid, where $g(U)$ is a continuous nondecreasing function of $U$, and $\nabla$ is the Laplace operator. Thus the theorems of the preceding sections insure the convergence of an iterative method for the three boundary value problems for this difference equation. In particular, since the functions $g_{i}(x)$ may be constant, we have this result for the Neumann and mixed problems for the Laplace and Poisson difference equations on a rectangular grid. The Dirichlet problem for the Laplace equation for such grids was treated by Diaz and Roberts [2], and their observation that this method provides an upper and lower bound on the solution at any step in the iteration now applies to the general case since we have shown that upper and lower functions can always be constructed. These upper and lower bounds on the solution are provided by applying the iterative procedure outlined in Sec. 5 . to the upper and lower functions as well as to the trial solution, and so it is useful to be able to give such functions in closed form. To do so for the Dirichlet problem for the Laplace difference equation is trivial [6]. The following theorem shows that a closed form can also be given for the Neumann problem for the Laplace or Poisson difference equation.

Theorem 6.1. Let $Y$ be a node function defined on the node set $P$ of a network $N$, 
and $F$ a node function defined on the boundary $B$. Let $q=\max \left(Q\left(B, P_{i}\right), P_{i} \varepsilon P\right)$, and $K=\max \left(0\left(P_{i}\right)\right.$, where $0\left(P_{i}\right)=$ (number of segments incident on $P_{i}$ minus one). Define the following quantities

$$
\begin{aligned}
H_{i} & =\sum_{i=0}^{i-1}(K)^{i}, \\
U & =\operatorname{trial} \text { function on } P \text { with respect to } F, \\
U^{\prime} & =\max \left(U_{i}, i=1,2, \cdots, n\right), \\
M^{\prime} & =\max \left(\left|y_{1}\right|,\left|y_{2}\right|, \cdots,\left|y_{n}\right|, U^{\prime}\right), \\
M & =M^{\prime}+F^{\prime}, \text { where } F=\max \left(F_{i}\right) .
\end{aligned}
$$

Then for the node function $W$ defined on $P$ by

$$
\begin{aligned}
& W_{i}=F_{i}, \quad P_{i} \varepsilon B, \\
& W_{i}=F^{\prime}+M \sum_{i=q+1} \sum_{-Q\left(B, P_{i}\right)}^{a} H_{i}, \quad P_{i} \varepsilon(P-B),
\end{aligned}
$$

we have

$$
\sum_{i=1}^{n}\left(W_{i}-W_{i}\right) \leq Y_{i}, \quad P_{i} \varepsilon(P-B)
$$

Further, $W \geq U$.

Proof. To show $W \geq U$ we note that $Q\left(B, P_{i}\right)>Q\left(B, P_{i}\right)$ if and only if $W_{i}>W_{i}$. Thus if $P_{i}$ is such that $Q\left(B, P_{i}\right)=1$, we have for $P_{i}$ in $(P-B)$

$$
W_{i} \geq W_{i}=F^{\prime}+M H_{a} \geq M-\left|F^{\prime}\right|=M^{\prime} \geq U_{i} .
$$

Relation (6.8) is verified by direct substitution.

7. Appendix. It can be shown (see, for example, Saltzer [7]) that a necessary condition that the Neumann problem have a solution is that $\sum_{i=1}^{n} Y_{i}=0$, where the $Y_{i}$ are the constant values of the influx prescribed at each node. Suppose (2.6) holds at the nodes of $(P-B)$. Then if we let $W \equiv 1$ in (3.1) and write the remaining sum in two parts, we have

$$
\sum_{k, B}\left[\sum_{i, S} \epsilon_{k j} I_{i}\right]=-\sum_{k, P-B}\left[\sum_{i, S} \epsilon_{k j} I_{j}\right]=-\sum_{k, P-B} Y_{k}=\sum_{k, B} Y_{k} .
$$

But for the Neumann problem, $B$ is a single node, and (7.1) thus becomes the statement that (2.6) holds at this node.

\section{REFERENCES}

1. G. Birkhoff and J. B. Diaz, Non-linear network problems, Quart. Appl. Math. 13, 431-443 (1956)

2. J. B. Diaz and R. C. Roberts, On the numerical solution of the Dirichlet problem for Laplace's difference equation, Quart. Appl. Math. 9, 355-360 (1952)

3. F. Harary, The number of functional digraphs, Math. Annalen 138, 203-210 (1959)

4. R. J. Duffin, Non-linear networks IIA, Bull. Amer. Math. Soc. 53, 963-971 (1947)

5. J. L. Synge, The fundamental theorem of electrical networks, Quart. Appl. Math. 9, 113-127 (1951)

6. J. B. Diaz and R. C. Roberts, Upper and lower bounds for the numerical solution of the Dirichlet difference boundary value problem, J. Math. Phys. 31, 184-191 (1952)

7. C. Saltzer, Discrete potential theory for two-dimensional Laplace and Poisson difference equations, NACA TN 4086, Washington, D. C. (1958) 